\title{
Research Roundup
}

\section{Computing direction}

I nhibitory signals and overlapping dendrites are enough to allow the sensing of direction, say Seunghoon Lee and Z. Jimmy Zhou (University of Arkansas for Medical Sciences, Little Rock, AR). These signals allow starburst amacrine cells (SACs) to send signals when light is moving in one direction but not when it is moving in another direction.

Direction-selective ganglion cells (DSGCs) have been studied since the 1960s, but the source of their selectivity was only

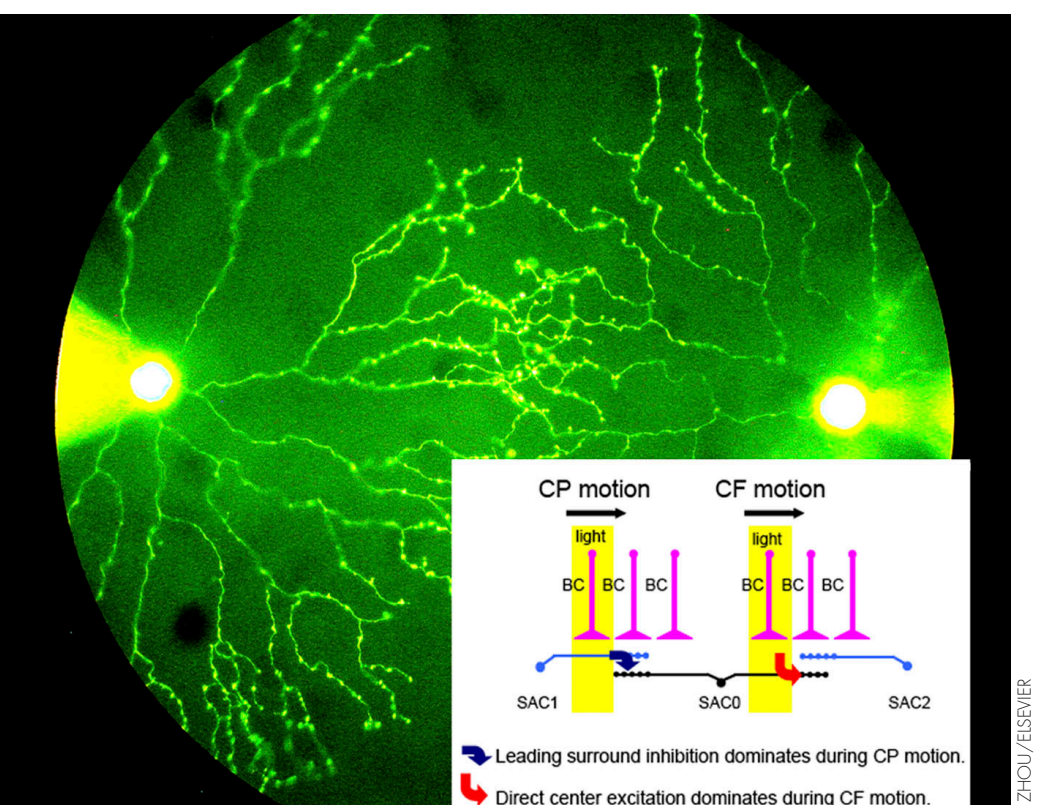

Starburst amacrine cells overlap to compute the direction that a light is moving. more recently defined as coming from SACs. Now, the Arkansas group has followed the activity of SACs in retinal preparations. Light going out along a SAC dendrite centrifugally (away from the cell body) resulted in SAC activation; the activated SAC then inhibits the downstream DSGC. But light coming in along a SAC dendrite centripetally activated the SAC far less, so there is little or no inhibition of the DSGC.

The centripetal inhibition of a SAC started when light was up to 2 dendritic radii away. This evidence, plus the transmitters involved and the presence of reciprocal inhibition, suggest that the source must be the neighboring, overlapping SACs. The group suggest that, as light moves toward a given SAC (SAC0), it first encounters the neighboring SAC (SAC1). SAC1 sends an inhibitory signal to $\mathrm{SAC} 0$, so by the time the light reaches SAC0 it is inhibited and barely reacts to the excitatory light signal. Consistent with this, the group saw inhibition of SACs by centripetal light before they saw (lowered) excitation. By contrast, light moving centrifugally first excites $\mathrm{SAC} 0$, and by the time it reaches neighboring SAC2 the game is over; any inhibition from SAC2 to SAC0 is irrelevant (because it is late) and reduced (because SAC0 has already inhibited SAC2).

SAC dendrites radiate in all directions, but each dendrite is essentially an independent unit for computing directionality. Thus, a single cell can contribute to detection of light coming from all possible directions. DSGCs, pointed along SAC dendrites in one direction, are turned on only when the SACs are not sending them inhibitory signals, which is when light is moving centripetally along the SAC dendrite. The next question is how this precise architecture is established in the first place. ЈСB Reference: Lee, S., and Z.J. Zhou. 2006. Neuron. 51:787-799.

\section{The}

Iiver that caveration after liver damage requires caveolin and lipid droplets, according to Manuel Fernández, Cecilia Albor (Universitat de Barcelona, Spain), Albert Pol, Robert Parton (University of Queensland, Brisbane, Australia), and colleagues. The results suggest that caveolin helps process or package storage lipids, and that the system somehow communicates with the cell cycle machinery.

Caveolin is best known as an essential component of ordered plasma membrane domains called caveolae. Besides overseeing signaling and sorting in caveolae, caveolin has also been seen around lipid droplets after partial hepatectomy. These lipid droplets have a core of storage lipids surrounded by a single monolayer of phospholipids. They are thought to bud
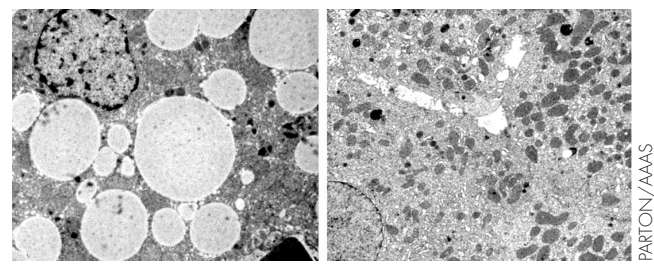

Lipid drops induced after partial hepatectomy (left) are missing in mice lacking caveolin (right). initiate recovery were activated, and levels of fatty acids increased in the serum and inside cells. But then things deteriorated. The signaling pathways persisted longer than normal, and lipid droplet accumulation and a marker of cell cycle progression were greatly reduced. The outcome was approximately half as much liver regeneration, and eightfold more death.

Glucose feeding rescued these cell cycle and survival defects. The glucose may be acting by providing an alternate source of energy, membrane lipids, or both.
Lipid droplet formation and caveolin's involvement in it remain a mystery. Perhaps caveolin helps package lipids in a way that allows them to be used properly. The group is now interested in mation and lipid metabolism are blocked in the mutants, and in teasing apart the link from lipid metabolism to cell cycle. JCB

Reference: Fernández, M.A., et al. 2006. Science. 313:1628-1632. seeing which stages of lipid droplet for- 